\title{
TABLE ERRATA
}

272.-H. M. Nautical Almanac Office, Planetary Coordinates, 1960-1980, H. M. Stationery Office, London, 1958.

P. 145, line 18; for $n^{3}$ read $n^{2}$.

P. 146, line 14; for 59.8 read 58.1 .

This erroneous value for $m$ is also on page xiv; the correct value brings the results of the example into still closer agreement with those on page xiii.

H. M. Nautical Almanac Office,

J. G. Porter Royal Greenwich Observatory, Hailsham, Sussex, England 